[0212-7199 (2004) 21: 11; pp 523-532] ANALES DE MEDICINA INTERN Copyright (C) 2004 ARAN EDICIONES, S.L.

AN. MED. INTERNA (Madric) Vol. 21, N. $^{\circ} 11$, pp. 523-532, 2004

\section{Estudio de la mortalidad y supervivencia en una cohorte de 1.115 pacientes con infección VIH (1989-97)}

\author{
F. J. SAN ANDRÉS REBOLLO, R. RUBIO GARCÍA' J. CASTILLA CATALÁN² \\ F. PULIDO ORTEGA', G. PALAO ${ }^{1}$, I. DE PEDRO ANDRÉS ${ }^{3}$, J. R. COSTA PÉREZ- \\ HERRERO $^{1}$, A. DEL PALACIO PÉREZ-MEDEL
}

Medicina Familiar y Comunitaria. Centro de Salud Potes. Madrid. ${ }^{1}$ Unidad VIH. Servicio de Medicina Interna. Hospital Universitario 12 de Octubre. Madrid. ${ }^{2}$ Centro Nacional de Epidemiología y Secretaría del Plan Nacional sobre Sida. Madrid. ${ }^{3}$ Medicina Familiar y Comunitaria. Centro de Salud Galve de Sorbe. Guadalajara
MORTALITY AND SURVIVAL IN A COHORT OF 1,115 HIV-INFECT ED PATIENTS (1989-97)

\section{ABSTRACT}

Background: To study survival and HIV/AIDS-related mortality from 1989 through 1997. To analyze the effect of antiretroviral treatment and prophylaxis against $\mathrm{P}$. carinii pneumonia (PCP-prophylaxis).

Patients and methods: We retrospectively studied a cohort of 1,115 HIV (+) outpatients (331 with AIDS-defining criteria) seen in our specific HIV hospital unit from January 1989 through May 1997. We analyzed the effect of different antiretroviral treatments on annual mortality rate. In survival studies we used Cox regression analysis to analyze survival over time as well as the effect of different opportunistic events, adherence and changes in treatment during follow up.

Results: Mortality rate was 13.7 per 100 person-years in 1994. It went down to 4.2 during the first half of 1997 ( $p=0.001)$. Mortality rate decreased depending on treatment received: $53 \%$ (CI 95=34\%-65\%) with monotherapy, 68\% (CI 95=38\%-84\%) with bitherapy, 86\% (CI 95=40\%-96\%) with triple therapy, and 49\% (CI =29\%-64\%) with PCPprophylaxis. Patients with more than $100 \mathrm{CD} 4$ had an increasing survival over time $(p=0.002)$. In AIDS patients good adherence to antiretroviral treatment and PCP-prophylaxis were associated with a lower risk of death $(R R=0.88 ; C I$ 95=0.63-1.22 and $R R=0.72 ; C I$ 95=0.55-0.95 respectively).

Conclusions: In recent years PCP-prophylaxis and antiretroviral treatment (especially combined therapy) have contributed to a decrease in AIDS-related mortality. Adherence to treatments relates to risk of death and survival.

KEY WORDS: Survivival. Mortality. Adherence. Compliant. Trend.

PALABRAS CLAVE: Supervivencia. Mortalidad. Adherencia. Cumplimiento. Tendencia.

San Andrés Rebollo FJ, Rubio García R, Castilla Catalán J, Pulido Ortega F, Palao G, de Pedro Andrés I, Costa Pérez-Herrero JR, del Palacio Pérez-Medel A. Estudio de la mortalidad y supervivencia en una cohorte de 1.115 pacientes con infección VIH (1989-97). An Med Interna (Madrid) 2004; 21:523-532.

\section{INTRODUCCIÓN}

Son muchos los estudios de supervivencia sobre los casos de sida diagnosticados durante las dos primeras décadas desde el inicio de esta epidemia. Sin embargo, la población objeto de estudio es en su mayoría homo/bisexual (1-15). En nuestro país, donde el colectivo más afectado por el VIH son los consumidores de drogas inyectadas (CDI) (16), son escasos los trabajos realizados sobre una muestra amplia de pacientes (17). Por otro lado, las modificaciones que han ido surgiendo en los criterios diagnósticos y ampliación de las enfermedades asociadas al sida, hacen necesario considerar el tipo de evento 
oportunista para valorar los cambios en el tiempo de la supervivencia, poder compararla con otras series y analizar sus factores determinantes $(4,7,18)$. Tampoco se han encontrado referentes en la literatura, previos a la instauración del tratamiento antirretroviral de gran actividad (TARGA), que hayan evaluado la adherencia a los tratamientos sobre la supervivencia y las tasas de mortalidad en amplios periodos de tiempo.

En una cohorte de pacientes con infección por el VIH se han analizado los siguientes aspectos: a) la tasa de mortalidad anual, su tendencia, y el impacto del tratamiento antirretroviral y de la profilaxis frente al Pneumocystis carinii (antiNPC); b) la supervivencia de los pacientes, sus factores pronósticos, la influencia de los distintos eventos oportunistas y de la adherencia a los tratamientos; y c) si la modificación del tratamiento antirretroviral durante el seguimiento contribuyó a mejorar su pronóstico.

\section{PACIENTES Y MÉTODOS}

\section{ÁMBITO Y POBLACIÓN DEL ESTUDIO}

Se revisaron retrospectivamente todas las historias clínicas de los pacientes con infección por el VIH mayores de 16 años que acudieron por primera vez a la Unidad VIH del Hospital General Universitario 12 de Octubre de Madrid entre enero de 1989 y diciembre de 1994 ( $n=2.011)$, y se incluyeron en el estudio aquellos que durante el periodo de observación (enero 1989-noviembre 1996): a) tuvieron un nivel medio de linfocitos CD4+ menor o igual a 500 células $/ \mathrm{mm}^{3}$ en dos determinaciones consecutivas, separadas por un intervalo mínimo de 3 meses; o b) cumplían criterios de sida. En el primer caso la fecha de incorporación a la cohorte fue la del primer recuento $\leq 500$ CD4 y para los pacientes con sida la fecha de su primera visita a la Unidad. El final del seguimiento correspondió a la fecha de la última observación (pérdida, muerte) o cierre del estudio (mayo-97). Se consideró pérdida, cuando no se disponía de datos más allá de los 3 meses anteriores a la fecha de cierre del estudio, y el paciente no había fallecido. Del total de pacientes atendidos en la unidad se excluyeron del estudio a 896: el 55\% de ellos por tener un seguimiento inferior a 6 meses, el $11 \%$ por no acudir al menos a la mitad de las visitas programadas, el $20 \%$ por no disponer de una historia clínica completa, y el $14 \%$ por tener estadio A o B con más de 500 CD $4+/ \mathrm{mm}^{3}$.

En relación con la primera visita se recogió la fecha de nacimiento, el sexo, la categoría de transmisión, el recuento de linfocitos CD4 $\left(\mathrm{n} / \mathrm{mm}^{3}\right)$ y las enfermedades previas diagnósticas de sida. En las visitas sucesivas (cada 4-6 meses según se tratara de paciente con sida o no) se recogió información de linfocitos CD4, tratamiento antirretroviral y profilaxis anti-NPC. Se consideró el recuento linfocitos CD4 más cercano a la fecha de la visita (control) dentro de \pm 3 meses, priorizando la determinación previa a la visita. Los regímenes de tratamiento antirretroviral que se consideraron fueron: monoterapia con zidovudina (ZDV), didanosida (DDI) o zalcitabina (DDC); biterapia o combinación de dos análogos de nucleósidos (ZDV, DDI, DDC, 3TC y D4T); y triterapia o combinación de dos análogos de nucleósidos más un inhibidor de la proteasa. Un paciente era asignado a un tipo de tratamiento en un semestre si lo había tomado al menos durante 2 meses.
La adherencia a los tratamientos se valoró según la autoestimación del cumplimiento del paciente (19). La adherencia en cada visita se consideró buena, cuando en la historia clínica no consta el incumplimiento de las indicaciones prescritas; regular, cuando el tratamiento se ha interrumpido por un periodo inferior a 3 meses, o las dosis empleadas son inferiores a las recomendadas; y mala, en la historia clínica consta que el paciente no sigue el tratamiento, o su interrupción es superior a tres meses. Se consideró que la adherencia al tratamiento antirretroviral y a la profilaxis anti-NPC se habían realizado correctamente durante el año cuando en los controles de ese año la adherencia había sido buena, o como máximo en uno de ellos fue regular. El cumplimiento del tratamiento antirretroviral y de la profilaxis anti-NPC a lo largo del periodo de seguimiento se consideraron realizadas correctamente si en más del $80 \%$ de los controles efectuados en ese periodo la adherencia fue buena.

\section{ANÁLISIS ESTADÍSTICO}

\section{ANÁLISIS DE LA MORTALIDAD}

Las tasas de mortalidad se calcularon como número de muertes por 100 pacientes/año. La tendencia en las tasas de mortalidad durante el periodo 1994-1997 se analizaron mediante técnicas de regresión de Poisson (20), ajustando por la edad ( $\leq 30$ años y > 30 años), el sexo, la categoría de transmisión, el estadio clínico (A-B y C), los linfocitos CD4 ( $\leq 100$ $\mathrm{y}>100$ ), y la adherencia al tratamiento y a la profilaxis anti$N P C$. En este mismo periodo se comparó el riesgo de muerte entre los pacientes que tomaron antirretrovirales respecto a aquellos que no siguieron un tratamiento específico, para lo cual se introdujo el régimen de tratamiento en un modelo de Poisson. Los resultados se ofrecen en términos de razón de riesgos (HR) junto a sus intervalos de confianza con un nivel del 95\% (IC95). Se efectuó el diagnóstico de colinealidad.

\section{ANÁLISIS DE SUPERVIVENCIA}

Las probabilidades de supervivencia se analizaron con el método de Kaplan-Meier. Las curvas de supervivencia se compararon mediante la prueba de Mantel-Haenszel (logrank) y el test exacto de Wilcoxon (Gehan). Se utilizaron modelos de riesgos proporcionales de Cox para calcular el riesgo de muerte asociado al primer evento diagnóstico de sida y a las enfermedades que se dieron en los pacientes con sida, ajustando por año del diagnóstico, diagnóstico previo o posterior a la incorporación en el estudio, linfocitos CD4, edad, sexo, categoría de transmisión y cumplimiento del tratamiento antirretroviral y de la profilaxis anti-NPC. Los resultados se ofrecen en términos de riesgo relativo (RR) junto a sus IC 95\%.

\section{Tendencia de la supervivencia en la cohorte}

Para analizar si la supervivencia de los pacientes (estadios A, B o C) se había modificado a lo largo del tiempo, se introdujo en un modelo de Cox el año en el que los pacientes habían iniciado el tratamiento antirretroviral, y se ajustó también por la edad, el estadio clínico, los CD4 y la adherencia al 
tratamiento. El sexo y la categoría de transmisión se excluyeron por no influir significativamente en las estimaciones.

\section{Análisis por intención de tratar: efecto de recibir y modificar el tratamiento antirretroviral sobre la supervivencia}

El tratamiento fue modelado como variable tiempo-dependiente (21) que toma el valor 0 mientras el paciente no recibe tratamiento y 1 cuando lo inicia, permaneciendo en esta situación aunque interrumpa o modifique la medicación. También se incluyeron como variables tiempo-dependientes la edad, el estadio clínico y los CD4. El sexo y la categoría de transmisión se introdujeron como variables independientes. Analizamos si el riesgo de muerte en aquellos que iniciaron tratamiento con ZDV se modificó cuando cambiaban a monoterapia con DDI/DDC, o a biterapia (ZDV+DD/DDC o 3TC+D4T). El tratamiento, el estadio clínico, los CD4, la edad y la profilaxis anti-NPC se incorporaron al modelo como variables tiempo-dependientes, mientras que el año de inicio de la ZDV se introdujo como variable independiente.

Los cálculos estadísticos se han realizado con los paquetes estadísticos SPSS 7.5 para Windows y PRESTA/PC V.2.2.
Para todos los resultados el nivel de significación se asume en $\mathrm{p} \leq 0,05$.

\section{RESULTADOS}

\section{CARACTERÍSTICAS DE LA COHORTE}

La cohorte está formada por 1.115 pacientes, cuyas características clínico-epidemiológicas al inicio del seguimiento se describen en la tabla I. El 25\% abandonaron el estudio, principalmente entre 1993 y 1996 (80\% de los casos) y el 33\% de los pacientes fallecieron por causas relacionadas con el sida. Hasta el primer semestre de 1993, la ZDV fue el principal fármaco utilizado. A partir de esa fecha, se incorporaron al tratamiento el DDI y el DDC y se fue instaurando progresivamente la biterapia, pasando del 3,9\% de las prescripciones a finales del 1994 al 46,7\% a mediados del 1996. En el primer semestre de 1997 la monoterapia suponía el 14,9\% de las prescripciones, la biterapia el 49,5\%, la triple terapia el 35,5\% y los inhibidores de la proteasa se utilizaba en el $44,3 \%$ de los tratamientos.

En las tablas II y III se comparan las características iniciales de los pacientes según el objetivo del análisis de supervivencia.

TABLA I

CARACTERÍSTICAS CLÍNICO-EPIDEMIOLÓGICAS DE LA COHORTE AL INICIO DEL SEGUIMIENTO (n=1.115)

\begin{tabular}{|c|c|c|c|}
\hline Características & $n$ & \multicolumn{2}{|c|}{ (IC 95\%) } \\
\hline Edad & & \multicolumn{2}{|c|}{$\begin{array}{c}\bar{x}=31,1(30,1-30,9) \\
\operatorname{rango}=17-78\end{array}$} \\
\hline $\begin{array}{l}\text { Varón } \\
\text { Mujer }\end{array}$ & $\begin{array}{l}842 \\
273 \\
\end{array}$ & $\begin{array}{l}75,5 \% \\
24,5 \% \\
\end{array}$ & $\begin{array}{l}(73 \%-78 \%) \\
(22 \%-27 \%) \\
\end{array}$ \\
\hline $\begin{array}{l}\text { Categoría de transmisión } \\
\text { Consumidores de drogas inyectadas } \\
\text { Heterosexual } \\
\text { Homosexual/bisexual } \\
\text { Otras }\end{array}$ & $\begin{array}{r}792 \\
150 \\
130 \\
43\end{array}$ & $\begin{array}{r}71,0 \% \\
13,5 \% \\
11,7 \% \\
3,9 \%\end{array}$ & $\begin{array}{c}(68,4 \%-73,7 \%) \\
(11,4 \%-15,5 \%) \\
(9,8 \%-13,5 \%) \\
(2,7 \%-5 \%)\end{array}$ \\
\hline $\begin{array}{l}\text { Estadio clínico } \\
\text { A o B } \\
\text { C }\end{array}$ & $\begin{array}{l}784 \\
331 \\
\end{array}$ & $\begin{array}{l}70,3 \% \\
29,7 \%\end{array}$ & $\begin{array}{l}(67 \mid 3 \%-73 \%) \\
(27 \%-32,3 \%)\end{array}$ \\
\hline $\begin{array}{l}\text { Enfermedad diagnóstica de sida }{ }^{1} \\
\text { Tuberculosis pulmonar y extrapulmonar } \\
\text { Candidiasis esofágica } \\
\text { NPC } \\
\text { Toxoplasmosis cerebral } \\
\text { Sarcoma de Kaposi } \\
\end{array}$ & $\begin{array}{r}331 \\
154 \\
42 \\
74 \\
19 \\
15\end{array}$ & $\begin{array}{r}4615 \% \\
12,7 \% \\
22,4 \% \\
517 \% \\
4,5 \% \\
\end{array}$ & $\begin{array}{c}(41,1 \%-51,9 \%) \\
(9,1 \%-16,3 \%) \\
(17,9 \%-26,8 \%) \\
(3,2 \%-8,2 \%) \\
(2,3 \%-6,8 \%) \\
\end{array}$ \\
\hline $\begin{array}{l}\text { Serología } \\
\text { IgG CMV } \\
\text { IgG Toxoplasma gondii }\end{array}$ & $\begin{array}{l}1013 \\
1.018 \\
\end{array}$ & $\begin{array}{l}90,1 \% \\
41,4 \% \\
\end{array}$ & $\begin{array}{c}(88,3 \%-93 \%) \\
(38,4 \%-44,5 \%)\end{array}$ \\
\hline Mantoux positivo & 947 & $21,9 \%$ & $(19,3 \%-24,5 \%)$ \\
\hline $\begin{array}{l}\text { Linfocitos CD4 }\left(\mathrm{n}^{\circ} / \mathrm{mm}^{3}\right) \\
\text { PacientesVIH (estadio A o B) } \\
\text { Pacientes SIDA } \\
\text { Total muestra }\end{array}$ & $\begin{array}{r}761 \\
321 \\
1.080\end{array}$ & $\begin{array}{r}\text { Mediana } \\
294 \\
100 \\
255\end{array}$ & $\begin{array}{c}(P 25-P 75) \\
(180-395) \\
(49,5-273) \\
(75-380)\end{array}$ \\
\hline
\end{tabular}

${ }^{1}$ Se incluyen las 5 enfermedades más frecuentes. Un mismo paciente puede debutar con más de un evento. 
TABLA II

TENDENCIA DE LA SUPERVIVENCIA EN LA COHORTE. CARACTERÍSTICAS CLÍNICO-EPIDEMIOLÓGICAS DE LOS PACIENTES SEGÚN EL AÑO EN EL QUE INICIAN EL TRATAMIENTO ANTIRRETROVIRAL

\begin{tabular}{|c|c|c|c|c|c|}
\hline Características & $\begin{array}{l}1989-91 \\
(n=237)\end{array}$ & $\begin{array}{c}1992 \\
(n=255)\end{array}$ & $\begin{array}{c}1993 \\
(n=224)\end{array}$ & $\begin{array}{c}1994 \\
(n=234)\end{array}$ & $p^{1}$ \\
\hline Linfocitos CD4 $\left\{\begin{array}{l}\text { Mediana } \\
\mathrm{P}_{25}-\mathrm{P}_{75}\end{array}\right.$ & $\begin{array}{c}195,5 \\
88,7-341,7 \\
\end{array}$ & $\begin{array}{c}220 \\
99-345 \\
\end{array}$ & $\begin{array}{c}238 \\
89-348 \\
\end{array}$ & $\begin{array}{c}228 \\
94-374 \\
\end{array}$ & ns \\
\hline $\begin{array}{l}\text { Estadio clínico } \\
\text { A o B } \\
\text { Sida }\end{array}$ & $\begin{array}{l}53,2 \% \\
46,8 \%\end{array}$ & $\begin{array}{l}64,7 \% \\
35,3 \%\end{array}$ & $\begin{array}{l}67,9 \% \\
32,1 \%\end{array}$ & $\begin{array}{l}70,1 \% \\
29,9 \%\end{array}$ & $p=0,001$ \\
\hline $\begin{array}{l}\text { Edad } \\
\quad \leq 30 \text { años } \\
>30 \text { años }\end{array}$ & $\begin{array}{l}52,7 \% \\
47,3 \% \\
\end{array}$ & $\begin{array}{l}53,4 \% \\
46,6 \% \\
\end{array}$ & $\begin{array}{l}53,9 \% \\
46,2 \% \\
\end{array}$ & $\begin{array}{l}37,2 \% \\
62,8 \% \\
\end{array}$ & $p<0,001$ \\
\hline $\begin{array}{l}\text { Sexo } \\
\quad \text { Varón } \\
\text { Mujer }\end{array}$ & $\begin{array}{l}77,2 \% \\
22,8 \%\end{array}$ & $\begin{array}{l}80,0 \% \\
20,0 \%\end{array}$ & $\begin{array}{l}71,0 \% \\
29,0 \%\end{array}$ & $\begin{array}{l}75,6 \% \\
24,4 \%\end{array}$ & ns \\
\hline $\begin{array}{l}\text { Categoría de transmisión } \\
\text { CDI } \\
\text { Heterosexual } \\
\text { Homosexual/bisexual }\end{array}$ & $\begin{array}{l}73,4 \% \\
10,5 \% \\
16,2 \%\end{array}$ & $\begin{array}{l}75,4 \% \\
11,1 \% \\
13,5 \%\end{array}$ & $\begin{array}{l}74,5 \% \\
13,9 \% \\
11,6 \%\end{array}$ & $\begin{array}{l}67,8 \% \\
19,8 \% \\
12,3 \%\end{array}$ & $p=0,056$ \\
\hline Cumplimiento del tratamiento antirretroviral & $210 \%$ & $28,8 \%$ & $25,3 \%$ & $24,9 \%$ & ns \\
\hline $\begin{array}{l}\text { Calidad del seguimiento } \\
\text { Pérdidas } \\
\text { Duración total (mediana) en meses } \\
\text { Tiempos censurados }\end{array}$ & $\begin{array}{l}28,7 \% \\
31,0 \\
44,1\end{array}$ & $\begin{array}{c}22,4 \% \\
38,0 \\
55,0\end{array}$ & $\begin{array}{c}24,1 \% \\
33,5 \\
41,5\end{array}$ & $\begin{array}{l}18,8 \% \\
29,5 \\
31,5\end{array}$ & $\begin{array}{l}\text { ns } \\
- \\
-\end{array}$ \\
\hline
\end{tabular}

${ }^{\top}$ Test Chi-cuadrado para variables cualitativas y Kruska-Wallis para las cuantitativas. NS: no significativo.

TABLA III

EFECTO DE RECIBIR Y MODIFICAR EL TRATAMIENTO ANTIRRETROVIRAL SOBRE LA SUPERVIVENCIA (ANÁLISIS POR INTENCIÓN DE TRATAR). CARACTERÍSTICAS CLÍNICO-EPIDEMIOLÓGICAS DE LOS PACIENTES AL INICIO DEL ESTUDIO

\begin{tabular}{|c|c|c|c|c|c|c|c|}
\hline \multirow[b]{2}{*}{ Características } & \multicolumn{4}{|c|}{ Han recibido tratamiento antirretroviral } & \multicolumn{3}{|c|}{ Modificación del tratamiento } \\
\hline & $\begin{array}{c}\text { No } \\
(n=79)\end{array}$ & $\begin{array}{c}S i \\
(n=1036)\end{array}$ & $p^{2}$ & $\begin{array}{c}\text { Continúan con ZDV } \\
(n=416)\end{array}$ & $\begin{array}{c}\text { Cambian a DDI, DDC } \\
(n-222)\end{array}$ & $\begin{array}{c}\text { Cambian a biterapia } \\
(n=103)\end{array}$ & ia \\
\hline Linfocitos CD4 $\left\{\begin{array}{c}\text { Mediana } \\
\text { P25_p75 }\end{array}\right.$ & $214,2-437,2$ & $96-357$ & $p$ & $69-324$ & $44,2-322$ & $119-388$ & $p=0,002$ \\
\hline $\begin{array}{l}\text { Estadio clínico } \\
\text { A o B } \\
\text { Sida }\end{array}$ & $\begin{array}{l}74,7 \% \\
25,3 \% \\
\end{array}$ & $\begin{array}{l}64,5 \% \\
35,5 \% \\
\end{array}$ & ns & $\begin{array}{l}51,0 \% \\
49,0 \% \\
\end{array}$ & $\begin{array}{l}64,3 \% \\
35,7 \% \\
\end{array}$ & $\begin{array}{l}70,5 \% \\
29,5 \% \\
\end{array}$ & $p<0,001$ \\
\hline $\begin{array}{l}\text { Edad } \\
\quad<30 \text { años } \\
>30 \text { años }\end{array}$ & $\begin{array}{l}59,9 \% \\
40,5 \% \\
\end{array}$ & $\begin{array}{l}52,5 \% \\
47,5 \%\end{array}$ & ns & $\begin{array}{l}52,7 \% \\
47,3 \%\end{array}$ & $\begin{array}{l}31,6 \% \\
68,4 \%\end{array}$ & $\begin{array}{l}26,8 \% \\
73,2 \%\end{array}$ & $p<0,001$ \\
\hline $\begin{array}{l}\text { Sexo } \\
\text { Varón } \\
\text { Mujer }\end{array}$ & $\begin{array}{l}74,7 \% \\
25,3 \% \\
\end{array}$ & $\begin{array}{l}75,6 \% \\
24,4 \% \\
\end{array}$ & ns & $\begin{array}{l}79,1 \% \\
20,9 \% \\
\end{array}$ & $\begin{array}{l}73,7 \% \\
26,3 \% \\
\end{array}$ & $\begin{array}{l}73,2 \% \\
26,8 \% \\
\end{array}$ & ns \\
\hline $\begin{array}{l}\text { Categoría de transmisión } \\
\text { CDI } \\
\text { Heterosexual } \\
\text { Homosexual/bisexual }\end{array}$ & $\begin{array}{c}79,8 \% \\
10,1 \% \\
3,8 \%\end{array}$ & $\begin{array}{l}70,4 \% \\
13,7 \% \\
12,3 \%\end{array}$ & $p=0,058$ & $\begin{array}{c}74,0 \% \\
9,1 \% \\
11,8 \%\end{array}$ & $\begin{array}{c}68,1 \% \\
16,6 \% \\
129\end{array}$ & $\begin{array}{c}67,0 \% \\
15,2 \% \\
25\end{array}$ & $p=0,020$ \\
\hline $\begin{array}{l}\text { Calidad del seguimiento } \\
\text { Pérdidas } \\
\text { Duración total (mediana ) en meses } \\
\text { Tiempos censurados }\end{array}$ & $\begin{array}{c}53,2 \% \\
13,0 \\
14,0\end{array}$ & $\begin{array}{c}22,9 \% \\
31,0 \\
36,0\end{array}$ & $\begin{array}{c}p<0,001 \\
- \\
-\end{array}$ & $\begin{array}{c}36,5 \% \\
19,0 \\
19,0\end{array}$ & $\begin{array}{c}16,1 \% \\
21,0 \\
24,0\end{array}$ & $\begin{array}{l}7,1 \% \\
12,0 \\
12,0\end{array}$ & $\begin{array}{c}p<0,001 \\
- \\
-\end{array}$ \\
\hline
\end{tabular}

'Los datos corresponden al momento en el que se produce el cambio del tratamiento. Biterapia:ZDV+DDI (DDC) o 3TC+D4T.

${ }^{2}$ Test de Chi-cuadrado para variables cualitativas y U de Mann-Whitney y Kruskal-Wallis para las cuantitativas.NS: no significativo. 
Tasas de mortalidad: tendencia y efecto del tratamiento antirretroviral y de la profilaxis anti-NPC

En la figura 1 se describe la evolución de la tasa de mortalidad anual. De 13,7 muertes por 100 personas-año en 1994 se pasó a 4,2 en los primeros 5 meses del 1997, lo que supone una reducción del 69\% $(\mathrm{p}=0,001)$.

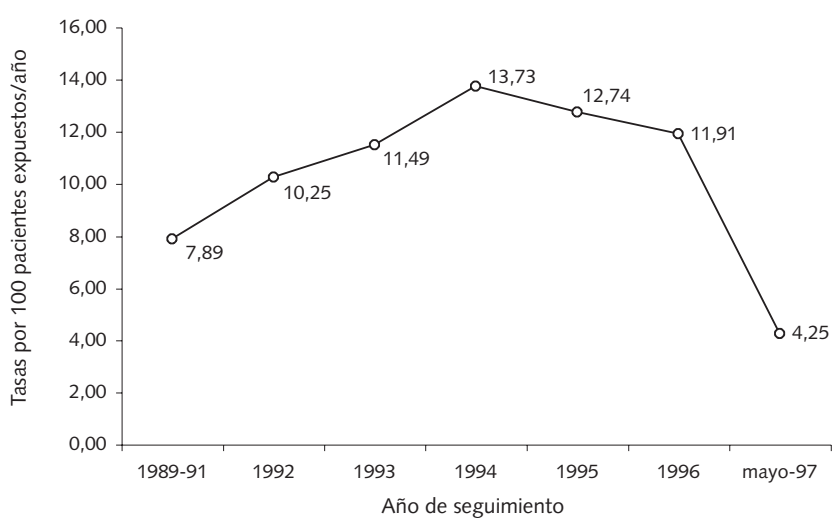

Fig. 1. Evolución de las tasas de mortalidad anuales por sida (19891997).

En el análisis multivariante el riesgo de muerte respecto al del 1994 fue de 0,95 (IC=0,69-1,32), 0,88 (IC=0,62-1,24) y 0,47 (0,21-1,03) para los pacientes seguidos en los años 1995, 1996 y primer semestre del 1997, respectivamente. Esta tendencia fue independiente de sexo, edad, categoría de transmisión, estadio clínico y profilaxis anti-NPC, pero no de los CD4, ya que en 1995 y 1996 sólo los sujetos con CD4 $>100 / \mathrm{mm}^{3}$ presentaron una disminución significativa del riesgo de muerte $(\mathrm{HR}=0,29 ; \mathrm{IC}=0,09-0,90$ y $\mathrm{HR}=0,31$; $\mathrm{IC}=0,10-0,96)$, mientras que en el 1997 la reducción fue independiente de los CD4.

En conjunto, los pacientes con buena adherencia al tratamiento antirretroviral y a la profilaxis anti-NPC presentaron un menor riesgo de muerte $(\mathrm{HR}=0,47$; $\mathrm{IC}=0,37-0,67$ y $\mathrm{HR}=$
$0,51$; IC $=0,36-0,71)$. Respecto a los pacientes que no siguieron tratamiento, la monoterapia se asoció a una disminución de la mortalidad del 53\% (IC=34\%-65\%), la biterapia del $68 \%(\mathrm{IC}=38 \%-84 \%)$ y la triple terapia del $86 \%(\mathrm{IC}=40 \%$ 96\%) (Tabla IV).

\section{Supervivencia}

El 56\% de los sujetos con sida murieron tras un seguimiento con una mediana de 17 meses (rango, 9-29 meses). La mediana de supervivencia fue de 23 meses $(I C=21-25 ; n=512)$ y la probabilidad acumulada de supervivencia al año del $75,1 \%$ (IC=71,2\%-78,9\%), a los 2 años del 49,9\% $(\mathrm{IC}=45,2 \%-54,7 \%)$ y al tercer año del $33,5 \%$ (IC=28,6\%$38,3 \%$ ) (Fig. 2). Las pérdidas de seguimiento ascendieron al $16,2 \%$. La supervivencia al diagnóstico de sida se asoció con la edad, por cada año de más al diagnóstico de sida la mortalidad se multiplica por 1,03 ( $\mathrm{IC}=1,01-1,04)$; con los linfocitos $\mathrm{CD} 4$, por cada incremento de 50 células/mm3 al momento del diagnóstico el riesgo de muerte disminuyó un $83 \%$ ( $\mathrm{IC}=82 \%$ $84 \%$ ); y el tipo de evento oportunista (Tabla V).

\section{Tendencia de la supervivencia en la cohorte}

La supervivencia global de los pacientes mejoró a medida que el tratamiento antirretroviral se había iniciado en años más recientes, pero sólo alcanzó la significación estadística en el grupo de sujetos con linfocitos CD4 $>100 / \mathrm{mm}^{3}$ al comienzo del tratamiento ( $R R=0,79 ; \mathrm{IC}=0,68-0,92)$ (Fig. 3), siendo la estimación de la supervivencia a los 36 meses del 70,3\% para los que inician los antirretrovirales en el periodo 1989-1991 y del 80,6\% para aquellos que lo hicieron en el 1994.

\section{Efecto del tratamiento antirretroviral y de la profilaxis anti-NPC sobre la supervivencia}

Los sujetos que iniciaron algún tratamiento antirretroviral tuvieron menor riesgo de morir que aquellos que nunca lo

TABLA IV

TASAS DE MORTALIDAD ${ }^{1}$ CON RELACIÓN AL TRATAMIENTO ANTIRRETROVIRAL Y AL PERIODO DE SEGUIMIENTO (1989-MAYO 1997)

\begin{tabular}{|c|c|c|c|c|c|c|c|c|c|c|c|c|c|}
\hline \multirow{2}{*}{\multicolumn{2}{|c|}{$\begin{array}{l}\text { Año y } \\
\text { semestres }\end{array}$}} & \multicolumn{3}{|c|}{ No tratamiento } & \multicolumn{3}{|c|}{ Monoterapia } & \multicolumn{3}{|c|}{ Biterapia } & \multicolumn{3}{|c|}{ Triterapia $^{2}$} \\
\hline & & $n^{3}$ & pac.año & tasas & & pac.año & tasas & & pac.año & tasas & & pac.año & tasas \\
\hline \multirow[t]{2}{*}{1994} & $1^{\circ}$ & 29 & 114,6 & 25,3 & 17 & 185,4 & 9,2 & 0 & 12,9 & 0,0 & - & - & - \\
\hline & $2^{\circ}$ & 25 & 119,2 & 21,0 & 17 & 202,7 & 8,4 & 1 & 13,3 & 7,520 & - & - & - \\
\hline \multirow[t]{2}{*}{1995} & $1^{\circ}$ & 20 & 114,2 & 17,5 & 16 & 187,0 & 8,6 & 1 & 26,9 & 3,7 & - & - & - \\
\hline & $2^{\circ}$ & 30 & 110,3 & 27,2 & 11 & 154,0 & 7,1 & 1 & 32,5 & 3,1 & 1 & 2,9 & 34,3 \\
\hline \multirow[t]{2}{*}{1996} & $1^{0}$ & 21 & 88,8 & 23,6 & 12 & 121,4 & 9,9 & 3 & 58,0 & 5,2 & 0 & 6,5 & 0,0 \\
\hline & $2^{\circ}$ & 18 & 68,3 & 26,3 & 4 & 74,6 & 5,4 & 4 & 93,3 & 4,3 & 0 & 9,5 & 0,0 \\
\hline 1997 & $1^{\circ}$ & 7 & 42,9 & 16,3 & 0 & 12,4 & 0,0 & 0 & 62,6 & 0,0 & 0 & 46,6 & 0,0 \\
\hline \multicolumn{2}{|c|}{ Total } & 150 & 659,3 & 228 & 77 & 937,5 & 8,2 & 10 & 299,5 & 3,3 & 1 & 66,5 & 1,5 \\
\hline
\end{tabular}

${ }^{1} \mathrm{~N}^{\circ}$ de muertes por 100 pacientes expuestos/año en cada una de las categorías de tratamiento y por semestre.

${ }^{2}$ Los inibidores de la proteasa son empleados en programas de tratamiento compasivo desde el $2^{\circ}$ semestre de 1995 , y en octubre de 1996 comienzan a formar parte de los esquemas de tratamiento.

${ }^{3}$ Número de muertes. Pacientes/año a riesgo. 


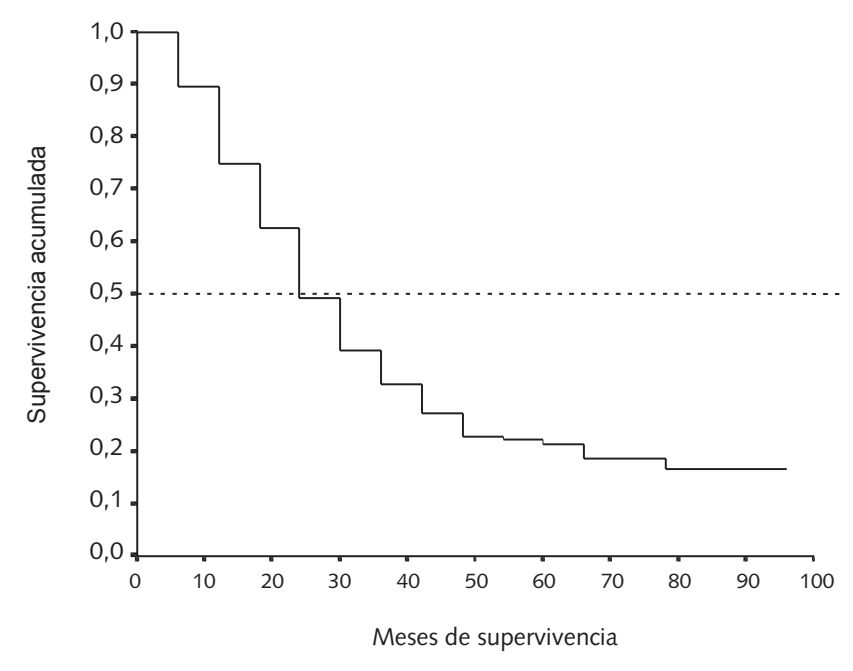

Fig. 2. Curva de supervivencia de los pacientes con sida ( $n=512)$.

hicieron $(\mathrm{RR}=0,40$; $\mathrm{IC}=0,20-0,81)$. Los pacientes con infección VIH asintomáticos que cumplieron adecuadamente con el tratamiento antirretroviral tuvieron un menor riesgo de muerte, sobreviviendo el $85,1 \%$ a los 3 años de seguimiento, frente al $61,9 \%$ en los no cumplidores $(\mathrm{RR}=0,43$; $\mathrm{IC}=0,29$ -
0,66). En los sujetos con sida, también se observa un efecto protector, pero no significativo $(\mathrm{RR}=0,88 ; \mathrm{IC}=0,63-1,22)$.

En los sujetos con infección VIH y CD $4<200 / \mathrm{mm}^{3}$ que cumplieron con la profilaxis anti-NPC se observó una disminución del riesgo de muerte no significativo $(\mathrm{RR}=0,95$; $\mathrm{IC}=$ $0,74-1,21 ; n=550)$, mientras que en los pacientes con sida la profilaxis se asoció a una reducción significativa del riesgo de morir $(\mathrm{RR}=0,72 ; \mathrm{IC}=0,55-0,95 ; \mathrm{n}=609)$.

\section{Efecto de modificar el tratamiento antirretroviral sobre la supervivencia}

Los pacientes tratados con ZDV que cambiaron a monoterapia con DDI/DDC o a biterapia tuvieron un menor riesgo de fallecer que los sujetos que continuaron con la $\mathrm{ZDV}$, aunque estadísticamente no significativos $(\mathrm{RR}=0,48 ; \mathrm{IC}=0,16-1,42$ y $\mathrm{RR}=0,29$; IC $=0,09-0,98)$.

\section{DISCUSIÓN}

\section{TASAS DE MORTALIDAD}

Nuestros resultados son congruentes con los informes procedentes de los sistemas de vigilancia epidemiológica del $\mathrm{VIH} /$ sida en el ámbito nacional (22) e internacional (23), don-

TABLA V

SUPERVIVENCIA Y RIESGO DE MUERTE EN PACIENTES CON SIDA, SEGÚN EL TIPO DE ENFERMEDAD OPORTUNISTA

\begin{tabular}{|c|c|c|c|c|c|c|c|c|c|c|c|c|c|c|c|c|c|c|}
\hline \multirow{3}{*}{ Enfermedades oportunlstas } & \multicolumn{9}{|c|}{ Corno primer evento } & \multicolumn{9}{|c|}{ Durante el seguimiento } \\
\hline & \multicolumn{4}{|c|}{$\begin{array}{l}\text { Supervivencia } \\
\text { (meses) }\end{array}$} & \multicolumn{3}{|c|}{$\begin{array}{l}\text { \%acumulado de } \\
\text { supervivenciaª }\end{array}$} & \multicolumn{2}{|c|}{$\begin{array}{l}\text { Riesgo de } \\
\text { muerte }^{3}\end{array}$} & \multicolumn{4}{|c|}{$\begin{array}{l}\text { Supervivencia } \\
\text { (meses) }\end{array}$} & \multicolumn{3}{|c|}{$\begin{array}{l}\text { \%acumulado de } \\
\text { supervivencia }\end{array}$} & \multicolumn{2}{|c|}{$\begin{array}{l}\text { Riesgo de } \\
\text { muerte }^{4}\end{array}$} \\
\hline & n & Md & $P_{75}$ & $P_{25}$ & $\tau 12$ & $\tau 24$ & $\tau 36$ & RR & $(\mid \mathrm{C} 95 \%)$ & n & Md & $P_{75}$ & $P_{25}$ & $\tau 12$ & $\tau 24$ & $\tau 36$ & RR & $($ (IC95\%) \\
\hline Tuberculosis pulmonar y extrapulmonar & 150 & 45 & 22 & - & 86,5 & 72,2 & 57,6 & 1,0 & - & 25 & 23. & 9 & 39 & 66,0 & 45,7 & 32,6 & 2,1 & $(1,0-4,3)$ \\
\hline Complejo demencia-sida & 4 & 30. & 2 & 30 & 71,4 & 71,4 & 0,0 & 1,4 & $(0,3-5,7)$ & 8 & 2 & 1 & 3 & $0,02 \mathrm{a}$ & - & - & 5,1 & $(1,5-17,9)$ \\
\hline Herpes muco-cutáneo y visceral & 7 & 21- & 5 & 39 & 66,7 & 44,4 & 44,4 & 1,5 & $(0,5-4,1)$ & 8 & 7 & 2 & 18 & 50,0 & 0,0 & - & 1,1 & $(0,1-8,8)$ \\
\hline Neumonía de repetición & 14 & 30 & 11 & - & 68,0 & 68,0 & 68,0 & 1,5 & $(0,5-4,2)$ & 6 & 18 & 9 & 21 & 63,6 & 21,2 & 21,2 & 1,7 & $(0,4-6,1)$ \\
\hline Candidiasis esofágica & 110 & 22. & 12 & 42 & 74,3 & 45,1 & 32,3 & 1,5 & $(1,0-2,3)$ & 78 & 11. & 6 & 20 & 50,7 & 16,2 & 9,0 & 2,5 & $(1,6-3,7)$ \\
\hline Neumonía por Pneumocystis carinii & 97 & 25. & 19 & 39 & 86,7 & 57,6 & 30,9 & 1,6 & $(1,1-2,4)$ & 49 & 7 & 2 & 19 & 41,3 & 17,7 & 11,8 & 2,2 & $(1,3-3,7)$ \\
\hline Mycobacterium avium complex & 10 & 25 & 8 & 32 & 66,7 & 53,3 & 38,1 & 1,7 & $(0,7-4,4)$ & 50 & 8 & 3 & 13 & 30,4 & 6,8 & 6,8 & 4,8 & $(2,8-8,3)$ \\
\hline Toxoplasmosis cerebral & 30 & 19 & 12. & 27 & 71,4 & 37,8 & 23,6 & 2,0 & $(1,2-3,5)$ & 24 & 8 & 4 & 12 & 24,4 & 17,5 & 8,7 & 12,5 & $(5,7-27,5)$ \\
\hline Enfermedad diseminada por CMV & 10 & 10 & 9 & 26 & 47,4 & 35,5 & 21,3 & 2,4 & $(1,1-5,4)$ & 15 & 5 & 3 & 10 & 17,2 & 0,0 & - & 4,3 & $(1,6-11,7)$ \\
\hline Retinitis por CMV & 23 & 15. & 6. & 22 & 62,8 & 20,9 & 9,0 & 2,5 & $(1,4-4,5)$ & 50 & 8 & 4 & 12 & 29,7 & 6,6 & 3,3 & 3,6 & $(2,3-5,4)$ \\
\hline Sarcoma de Kaposi & 24 & 23. & 12 & 29 & 77,3 & 42,9 & 21,5 & 2,7 & $(1,5-4,8)$ & 13 & 16 & 5 & 26 & 52,0 & 33,1 & 6,6 & 3,4 & $(1,4-7,9)$ \\
\hline Síndrome caquéctico & 11 & 17 & 4 & 23 & 70,0 & 19,1 & 19,1 & 2,9 & $(1,2-6,9)$ & 14 & 4 & 2 & 14 & 30,8 & 10,3 & 10,3 & 4,9 & $(2,0-11,7)$ \\
\hline Leucoencefalopatia multifocal progresiva & 13 & 17 & 3 & 22 & 65,2 & 26,1 & 26,1 & 3,3 & $(1,3-8,5)$ & 12 & 6 & 2 & 18 & 30,4 & 18,3 & 18,3 & 7,2 & $(3,2-16,2)$ \\
\hline Criptosporidiosis & 10 & 13 & 4. & 22 & 57,9 & 23,2 & 23,2 & 4,3 & $(1,7-9,5)$ & 18 & 5 & 2,0 & 15 & 27,3 & 5,4 & - & 5,5 & $(2,6-11,5)$ \\
\hline Criptococosis & 5 & 3 & 9 & 14 & 75,0 & 0,0 & 0,0 & 7,6 & $(2,3-25,2)$ & 9 & 8. & 4 & 11 & 25,0 & 0,0 & - & 4,6 & $(1,8-11,6)$ \\
\hline Linfoma cerebral primario & 5 & 2. & 4 & 8 & 20,0 & 0,0 & - & 8,2 & $(2,5-27,2)$ & 7 & 1 & 0,9 & 10 & 14,3 & 14,3 & 0,0 & 8,5 & $(1,7-42,9)$ \\
\hline Linfoma no Hodgkin & 18. & 5 & 2 & 22 & 37,1 & 24,8 & 12,4 & 11,1 & $(5,8-22,2)$ & 4 & $4 a$ & 2 & 8 & 0,02 & - & - & 26,1 & $(0,5-563,2)$ \\
\hline
\end{tabular}

${ }^{1}$ Pacientes $\mathrm{VIH}$ que desarrollaron sida durante el seguimiento $(\mathrm{n}=278)$ y los casos sida cuyo diagnóstico se efectuó en los 3 meses previos a su incorporación al estudio $(n=234)$. En un mismo paciente pueden coincidir más de una enfermedad como presentación inicial de sida.

${ }^{2}$ Proporción de sujetos que sobreviven un mínimo de 12,24 ó 36 meses. a) Los pacientes con complejo demencia- sida y linfoma no Hodgkin tienen una supervivencia a los 6 meses de 12,5 y de $42,9 \%$ respectivamente.

${ }^{3}$ Estimado a través de un modelo de Cox en el que se incluyen: edad y CD4 al diagnóstico de sida, cumplimiento del tratamiento antirretroviral y de la profilaxis anti-NPC, enfermedad oportunista, año de diagnóstico y si es previo o no a la entrada en el estudio. La tuberculosis se tomó como categoría de referencia.

${ }^{4}$ Estimado a través de un modelo de Cox con las mismas variables descritas previamente, pero los CD4 y el tipo de enfermedad oportunista se modelaron como variables tiempo-dependientes. RR= riesgo de muerte en los pacientes con sida que desarrollan un evento específico en su evolución, respecto a los sujetos que no la desarrollan. 


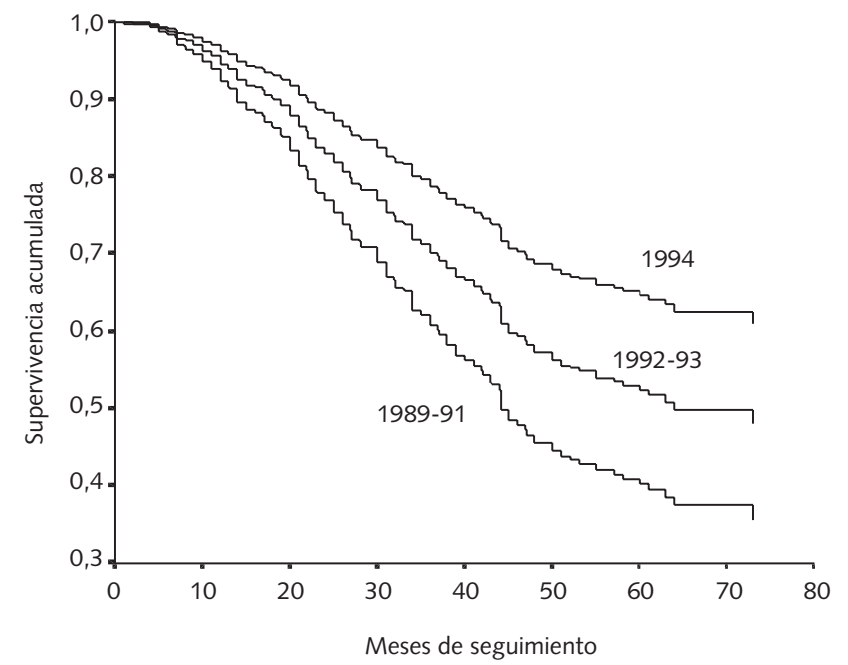

Fig. 3. Supervivencia en los pacientes con C4> 100/ $\mathrm{mm}^{3}$, según el año de inicio del tratamiento antirretroviral.

de se observan también descensos de la mortalidad a partir de 1994.

Esta tendencia se contrapone a lo esperable por la historia natural de la enfermedad, que debido al progresivo deterioro de la función inmune y envejecimiento de la cohorte llevaría a un incremento de la mortalidad. La disminución más acentuada del riesgo de muerte en los últimos años del seguimiento coincide con el incremento en la utilización del tratamiento combinado, como también han descrito otros autores (1012,24,25). La reducción del riesgo en los años 1995 y 1996 se produjo principalmente en los sujetos con CD4>100/ $\mathrm{mm}^{3}$, mientras que en 1997 el riesgo de muerte disminuyó de forma independiente a los CD4, lo que se explica por el cambio en la indicación del tratamiento antirretroviral, ya que el TARGA fue utilizado escasamente por el $5 \%$ de los pacientes en el periodo 95-96, frente al 36\% en los primeros meses del 97. Además, se ha constatado como el riesgo de muerte asociado a la triple terapia es mucho menor comparado con la mono o biterapia.

\section{Supervivencia de los pacientes con sida}

El patrón de supervivencia observado es similar al descrito en la literatura. La mediana de supervivencia de los sujetos con sida en la primera década de la epidemia oscilaba entre los 9,5 y 24 meses, y la probabilidad acumulada de supervivencia a los 3 años, entre el 13 y $32 \%(2,4,5,6,17,26,27)$. Estudios más recientes señalan una mediana entre 20 y 46 meses, alcanzando el porcentaje de pacientes que permanecen vivos a los dos años el 42\%-60\% (7,15,28-30).

En términos generales, la supervivencia para la mayor parte de los eventos diagnósticos de sida en la cohorte es superior a la descrita en otros estudios referidos a la primera década de los ochenta $(2,4,5,18,31,32)$, con la excepción del LNH y el $\mathrm{LCP}$, cuyos pronósticos prácticamente no han variado con el tiempo $(4,5,18,33-35)$ y la criptococosis y criptosporidiosis que en algunos trabajos se muestra superior $(18,36)$. La mediana de supervivencia para el MAC como enfermedad definitoria de sida casi triplica a la descrita en la literatura $(37,38)$, pero el pequeño tamaño de la muestra $(n=10)$ no permite extraer conclusiones. Por la misma razón no ha de tenerse muy en cuenta los datos con relación al complejo demencia-sida.

Cuando se analiza el riesgo de muerte asociado al desarrollo de las distintas enfermedades indicativas de sida, independientemente del momento de su presentación, nuestros resultados son equiparables a los descritos en la literatura (18,35,38-41), coincidiendo en señalar al LNH como uno de los procesos oportunistas de peor pronóstico (4,18,35,41-44).

Aunque algunos trabajos señalan diferencias en la supervivencia de los pacientes con sida respecto a la raza, el sexo y la categoría de transmisión, éstas pueden estar reflejando diferencias de acceso a los tratamientos y cuidados de salud, o en el patrón de enfermedades diagnósticas de sida $(15,44-46)$. Coincidiendo con nuestros datos, los factores que han demostrado mayor consistencia y que se asocian a un peor pronóstico son: una edad mayor al diagnóstico, un bajo nivel de linfocitos CD4 y el tipo de evento oportunista $(7,14,27,41-43,45)$

\section{Tendencia de la supervivencia en la cohorte}

La mejora de la supervivencia global para la subcohorte de pacientes que iniciaron el tratamiento antirretroviral en años más recientes puede estar relacionado con los cambios sucesivos en su manejo terapéutico, en particular la instauración de la terapia combinada, ya que tienen más probabilidades de haber recibido en su evolución estos tratamientos. Posiblemente esta circunstancia se ha dado principalmente en aquellos que a priori tenían un mejor pronóstico y justifique porqué el beneficio se ha observado únicamente en los pacientes que tenían el sistema inmunológico menos deteriorado. En este sentido varios estudios $(47,48)$ señalan que el cambio del tratamiento con ZDV a monoterapia con DDI/DDC o a biterapia mejoraba la supervivencia, pero en aquellos con más de 100 ó 150 linfocitos CD4/ $\mathrm{mm}^{3}$. No obstante, y a pesar del ajuste, parte de las diferencias que se observan pueden deberse también a la peor situación clínico-inmunológica de los pacientes que iniciaron el tratamiento en el periodo 1989-91.

\section{Efecto del tratamiento antirretroviral y de la profilaxis anti-NPC sobre la supervivencia}

En el análisis por intención de tratar, los sujetos que iniciaron algún tratamiento antirretroviral presentaron un riesgo menor de morir que aquellos que nunca lo hicieron. En el estudio sobre adherencia, el buen cumplimiento del tratamiento antirretroviral y de la profilaxis anti-NPC se asociaban a una reducción de la mortalidad en los sujetos con sida.

Numerosos estudios observacionales muestran que el uso de la monoterapia, principalmente con ZDV, mejora la supervivencia y disminuye la mortalidad de los pacientes con infección VIH/sida $(2,3,8,41,49,50)$, si bien en muchos de ellos el efecto probablemente esté sobrestimado debido al sesgo de selección (0). Algunos estudios más recientes señalan sólo un beneficio de la monoterapia en los sujetos con sida $(15,25)$, pero también los hay que la asocian a aumento de la mortalidad (51). Estas discrepancias pueden deberse a diferencias 
metodológicas con relación al tipo de estudio (cohortes o de base poblacional) y al criterio utilizado para evaluar la eficacia del tratamiento. Además, en muchos de los estudios publicados inicialmente, el tiempo de seguimiento no supera los 2 años, por lo que es más probable que en ellos se detecten un posible beneficio de la ZDV (efecto transitorio), pudiendo prolongar el tiempo libre de sida, pero no necesariamente aumentar o mejorar la supervivencia una vez que éste aparezca (52).

Posteriormente otros estudios también han descrito beneficios del tratamiento antirretroviral combinado, con reducciones en el riesgo de muerte del $28 \%$ al $76 \%$ para la biterapia y del $65 \%$ al $85 \%$ para la triple terapia $(15,25,51,53)$.

La mayor parte de los estudios señalan que la profilaxis anti-NPC disminuye el riesgo de muerte, oscilando esta reducción entre el $21 \%$ y el $52 \%(8,9,15,54)$. Destaca el artículo de Chaisson RE et al (9) por ser uno de los pocos trabajos en donde se analiza el efecto de la adherencia a los tratamientos sobre la mortalidad. Estos autores observaron una reducción del riesgo de muerte durante el seguimiento de más del $50 \%$ en los pacientes que recibieron profilaxis con pentamidina y cotrimoxazol.

\section{Efecto de modificar el tratamiento antirretroviral sobre la supervivencia}

Al igual que otros autores $(13,48,54-57)$, se ha observado una disminución del riesgo de muerte en los pacientes a los que se les modificó la medicación antirretroviral durante su evolución, aunque estadísticamente no significativo. Esta circunstancia podría estar relacionada con varios factores: a) la existencia de otras variables de difícil control que influyan sobre la supervivencia, como puedan ser aquellas que intervengan en la decisión del médico a la hora de modificar el tratamiento (57); b) el que más de un tercio de la cohorte esté constituida inicialmente por sujetos con sida, mientras que p. ej., en el trabajo de Graham NMH y cols. (54), donde sí se observa una reducción significativa en el riesgo de muerte para los sujetos que cambiaron a DDI/DDC o combinaron estos fármacos con la ZDV, ningún paciente tenía sida; c) el que se haya valorado conjuntamente al DDI y DDC, tanto en monoterapia como en biterapia, señalándose en diferentes ensayos clínicos un menor efecto del DDC $(48,56)$; y d) el fra- caso terapéutico es más probable en los sujetos previamente tratados con ZDV debido a la aparición de resistencias (55).

\section{Limitaciones y sesgos del estudio}

Los datos han sido recogidos retrospectivamente a partir de las historias clínicas. Para minimizar un posible sesgo de selección cuando se analiza la efectividad de un tratamiento sobre la supervivencia, se ha introducido en los modelos esta variable como tiempo-dependiente, y esta estrategia también se ha utilizado para valorar el riesgo de muerte en un individuo cuando desarrolla una determinada enfermedad oportunista en algún punto de su seguimiento (41). El cumplimiento del tratamiento se ha valorado mediante criterios operativos, y sólo el $21 \%$ de los pacientes cumplieron adecuadamente con la medicación antirretroviral, lo que contrasta con el $48 \%$ descrito por otros autores en una cohorte de características similares a la nuestra (58). Es posible que el punto de corte elegido haya sido demasiado exigente y deje fuera a pacientes que han tomado estos fármacos en algún momento de su seguimiento (cumplidores parciales) (59) estrechándose el posible margen de beneficio cuando se comparan ambos grupos. Aunque la tasa de abandonos es alta entre 1993 y 1996, lo que pude infraestimar las tasas de mortalidad en esos años, en la mayoría de los casos se trata de pacientes asintomáticos y con buena situación inmunológica cuando abandonaron el estudio. En el análisis sobre la tendencia de la supervivencia y en los análisis por intención de tratar las pérdidas fueron importantes por lo que deben tomarse con cautela los resultados, máxime cuando el estudio de las pérdidas reveló en alguno de ellos diferencias que sugieren una sobreestimación del efecto que se pretende cuantificar. La exclusión de los casos de sida con un seguimiento inferior a los 6 meses puede sobreestimar la supervivencia, sin embargo, cuando se incluyeron en el análisis los resultados no variaron significativamente.

En resumen, en una amplia cohorte de pacientes con infección por VIH seguida entre 1989 y 1997, se ha observado una disminución de las tasas de mortalidad, y un aumento en la supervivencia de los pacientes con sida, atribuible a la monoterapia y en mayor medida a la terapia combinada. Asimismo, la adherencia a los tratamientos se relaciona con una menor mortalidad y una mayor supervivencia.

\section{Bibliografía}

1. Hoover DR. The effects of long-term zidovudine therapy and Pneumocystis carinii prophylaxis on HIV disease: a review of the literature. Drugs 1995; 49: 20-36.

2. Lemp GF, Payne SF, Neal D, Temelso T, Rutherford GW. Survival trends for patients with AIDS. JAMA 1990; 263: 402-406.

3. Osmond D, Charlebois E, Lang W, Shiboski S, Moss A. Changes in AIDS survival time in two San Francisco cohorts of homosexual men, 1983 to1993. JAMA 1994; 271: 1.083-1.087.

4. Jacobson LP, Kirby AJ, Polk S, et al. Changes in survival after Acquired Immunodeficiency Syndrome (AIDS): 1984-1991. Am J Epidemiol 1993; 138: 952-964.

5. Blum S, Singh TP, Gibbons J, et al. Trends in survival among persons with Acquired Immunodeficiency Syndrome in New York City: the experience of first decade of the epidemic. Am J Epidemiol 1994; 139: 351-361.

6. Buira E, Gatell JM, Zamora L, Nallolas J, Miró JM, Soriano E. Análisis de 1.187 casos consecutivos de sida: variaciones y tendencias en el tiempo. Enferm Infecc Microbiol Clin 1996; 14: 290-295.

7. Mocroft A, Youle M, Morcineck J, et al, for the Royal Free/Chelsea and Westminster Hospitals Colaborative Group. Survival after diagnosis of AIDS: a prospective observational study of 2.625 patients. BMJ 1997; 314: 409-413.

8. Graham NMH, Zeger SL, Park LP, et al. The effects on survival of early treatment of Human Immunodeficiency Virus infection. N Engl J Med 1992; 326: 1.037-1.042. 
9. Chaisson RE, Keruly J, Richman DD, Moore RD, for the Zidovudine Epidemiology Group. Pneumocystis prophylaxis and survival in patients with advanced human immunodeficiency virus infection treated with zidovudine. Arch Intern Med 1992; 152: 2.009-2.013.

10. Palella FJ, Delaney KM, Moorman AC, et al, and the HIV Outpatient Study Investigators. Declining morbidity and mortality among patients with advanced human immunodeficiency virus infection. N Engl J Med 1998; 338: 853-860.

11. Detels R, Muñoz A, McFarlane G, et al, for the Multicenter AIDS Cohort Study investigators. Effectiveness of potent antiretroviral therapy on time to AIDS and death in men with known HIV infection duration. JAMA 1998; 280: 1.497-1.503.

12. Vittinghoff E, Scheer S, O’Malley P, Colfax G, Holmberg SD, Buchbinder SP. Combination antiretroviral therapy and recent declines in AIDS incidence and mortality. J Infect Dis 1999; 179: 717-720.

13. Hogg RS, Heath KV, Yip B, et al. Improved survival among HIV-infected individuals following initiation of antiretroviral therapy. JAMA 1998; 279: 450-454.

14. Moore RD, Keruly J, Richman DD, Creagh-Kirk T, Chaisson RE. Natural history of advanced HIV disease in patients treated with zidovudine. The Zidovudine Epidemiology Study Group. AIDS 1992; 6: 671-677.

15. McNaghten AD, Hanson DL, Jones JL, Dworkin MS, Ward JW, and the Adult/Adolescent Spectrum od Disease Group. Effects of antiretroviral therapy and opportunistic illness primary chemoprophylaxis on survival after AIDS diagnosis. AIDS 1999; 13: 1.687-1.695.

16. European Centre for the Epidemiological Monitoring of AIDS HIV/AIDS surveillance in Europe. End-year report 2000, n 65 2001.

17. Valero JA, Ruiz-Navarro MD. Evolución de la supervivencia del sida en España: efecto de la inclusión de la tuberculosis pulmonar en la definición de caso en la era de los inhibidores de la proteasa. Rev Clin Esp 1998; 198: 526-528.

18. Mocroft AJ, Lundgren JD, Monforte AD, et al, for the AIDS in Europe Study Group. Survival of AIDS patients according to type of AIDSdefinig event. Int J Epidemiol 1997; 28: 400-407.

19. Gil V, Pineda M, Martínez JL, Belda J, Santos ML, Merino J. Validez de 6 métodos indirectos para valorar el cumplimiento terapeútico en hipertensión arterial. Med Clin (Barc) 1994; 102: 532-536.

20. Abraira V, Pérez de Vargas A. Regresión de Poisson. En: Abraira V, Pérez de Vargas A, editores. Métodos multivariantes en bioestadística. Madrid: Centro de Estudios Ramón Areces; 1996. p. 263-281.

21. Kleinbaum DG. Extension of the Cox Proportional Hazards Model for Time-Dependent Variables. En: Dietz k, Gail M, Krickerberg K, Singer B, editores. Survival analysis: A self-learning text. New York: Springer-Verlag; 1997. p. 211-253.

22. Castillla J, De la Fuente, L. Evolución del número de personas infectadas por el VIH y de los casos de sida. España: 1980-1998. Med Clin(Barc) 2000; 115: 85-89.

23. Centre Européen pour la Surveillance Epidémiologique du Sida. Surveillance du VIH/sida en Europe: Rapport nº 61, 30 juin 1999.

24. Moore RD, Chaisson RE. Natural history of HIV infection in the era of combination antiretroviral therapy. AIDS 1999; 13: 1.9331.942 .

25. Egger M, Hirschel B, Francioli P, et al, and the Swiss HIV Cohort Study. Impact of new antiretroviral combination therapies in HIV infected patients in Swizerland: prospective multicentre study. BMJ 1997; 315: 1.194-1.199.

26. Rothenberg R, Woelfel M, Stoneburner R, Milberg J, Parker R, Truman B. Survival with the Acquired Immunodeficiency Syndrome: experience with 5.833 cases in New York City. N Engl J Med 1987; 317: 1.2971.302 .

27. Caylà JA, Artazcoz L, Iglesias B, Jansà JM, Plasència A. Epidemiología del sindrome de inmunodeficiencia adquirida en Barcelona (1981-1991) (II). Estudio de mortalidad y de supervivencia. Med Clin (Barc) 1994; 102: 129-135.

28. García de Olalla P, Caylà JA, Brugal MT, Galdós H, Jansá JM, Clos R. Evolución de la mortalidad y supervivencia del sida en Barcelona (1981-1997). Med Clin (Barc) 1999; 113: 169-170.

29. Schwarcz SK, Chin L, Vittinghoff E, Katz MH. Impact of protease inhibitors and other antiretroviral treatments on Acquired Immunodeficiency Syndrome survival in San Francisco, California; 1987-1996. Am J Epidemiol 2000; 152: 178-185.

30. Lee LM, Karon JM, Selik R, Neal J, Fleming PL. Survival after AIDS diagnosis in Adolescents and Adults during the Treatment era, United States, 1984-1997. JAMA 2001; 285: 1308-1315.
31. Miralles R, Garcés JM, Gallén M, et al. Síndrome de inmunodeficeincia adquirida: estudio descriptivo y análisis de la supervivencia en 73 casos. Med Clin (Barc) 1990; 94: 401-405.

32. Lundgren JD, Barton SE, Katlama C, et al, for the Multicenter Study Group on AIDS in Europe. Changes in survival over time after a first episode on Pneumocystis carinii for european patients with Acquired Immunodeficiency Syndrome. Arch Intern Med 1995; 155: 822-828.

33. Rubio R, Pulido F, Pintado V, et al. Linfomas no hodgkinianos asociados al síndrome de la inmunodeficiencia adquirida. Estudio clínico multicéntrico de 77 casos. Grupo Cooperativo sida y Tumores de Madrid. Med Clin (Barc) 1995; 104: 481-486.

34. Casabona J, Salas T, Salinas R. Trends and survival in AIDS-associated malignancies. Eur J Cancer 1993; 294: 877-881.

35. Conti S, Masocco M, Pezzotti P, et al. Differential impact of combined antiretroviral therapy on the survival of italian patients with specific AIDS-defining illnesses. J Acquir Immune Defic Syndr 200; 25: 451458.

36. Luo K, Law M, Kaldor JM, McDonald, Cooper D. The role of initial AIDS-defining illness in survival following AIDS. AIDS 1995; 9: 57 63.

37. Herrero JA, Sánchez MD, Palenque E, et al. Infección diseminada por Mycobacterium avium-intracellulare en pacientes con sida. Med Clin (Barc) 1993; 100: 171-173.

38. Low N, Pfluger D, Egger M, and the Swiss HIV Cohort Study. Disseminated Mycobacterium avium complex disease in the Swiss HIV Cohort Study: increasing incidence, unchanged prognosis. AIDS 1997; 11: 1.165-1.171

39. Oksenhendler E, Carreau I, Tournerie C, Azihary M, Carbon C, Aboulker JP. Toxoplasma gondii infection in advanced HIV infection. AIDS 1994; 8: 483-487.

40. Gallant JE, Moor RD, Richman DD, Keruly J, Chaisson RE. Incidence and natural history of cytomegalovirus disease in patients with advanced human immunodeficiency virus disease treated with zidovudine. The Zidovudine Epidemiology Study Group. J Infect Dis 1992; 166: 1.223-1.227

41. Petruckevitch A, Del Amo J, Phillips AN, et al. Disease progression and survival following specific AIDS-defining conditions: a retrospective cohort study of 2.048 HIV-infected persons in London. AIDS 1998; 12 : 1.007-1.013.

42. Chaisson RE, Gallant JE, Keruly JC, Moore RD. Impact of opportunistic disease on survival in patients with HIV infection. AIDS 1998; 12 29-33.

43. Casari S, Donisi A, Paraninfo G, et al. Pronostic factors correlated with survival in AIDS patients. Eur J Epidemiol 1999; 15: 691-698.

44. Sha BE, Benson CA, Pottage JC, Urbanski PA, Daugherty SR, Kessler HA. HIV infection in woman: an observational study of clinical characteristics, disease progression, and survival for a cohort of woman in Chicago. J Acquir Immune Defic Syndr Hum Retrovirol 1995; 8: 486495.

45. Mocroft A, Johnson MA, Phillips AN. Factors affecting survival in patients with the acquired immunodeficiency syndrome. AIDS 1996; 10: $1.057-1.065$.

46. Prins M, Veugelers PJ, for the European Seroconverter Study and Tricontinental Seroconverter Study. Comparison of progression and nonprogression in injecting druf users and homosexual men with documented dates of HIV-1 seroconversion. AIDS 1997; 11: 621-631.

47. Gatell JM, González-Lahoz J, Clotet B, et al, and the ddI Iberian Study Group. Switching from zidovudine to didanosine in patients with symptomatic HIV infection no disease progression. J Acquir Immune Defic Syndr Hum Retrovirol 1996; 12: 249-258.

48. Fischl MA, Stanley K, Collier AC, et al, and the NIAID AIDS Trials Group. Combination and monotherapy with zidovudine and zalcitabine in patients with advanced HIV disease. Ann Intern Med 1995; 122: 24 32.

49. Lundgren JD, Phillips AN, Pedersen C, et al, for the AIDS in Europe Study Group. Comparison of long-term prognosis of patients with AIDS treated and not treated with zidovudine. JAMA 1994; 271: 1.088 1.092 .

50. Buira E, Gatell JM, Miró JM, et al. Influence of treatment with zidovudine (ZDV) on the long-term survival of AIDS patients. J Acquir Immune Defic Syndr 1992; 5: 737-742.

51. Pezzotti P, Napoli PA, Acciani S, et al. Increasing survival time after AIDS in Italy: the role of new combination antiretroviral therapies. AIDS 1999; 13: 249-255. 
52. Hamilton JD, Hartigan PM, Simberkoff MS, et al, and the Veterans Affairs Cooperative Study Group on AIDS Treatment. A controlled trial of early versus late treatment with zidovudine in symptomatic Human Immunodeficiency Virus infection. N Eng J Med 1992; 326: 437-443.

53. Gálvez C, Blanco F, del Amo J, Ruiz-Navarro MD, Soriano V, González J. Efecto del tratamiento antirretrovírico y de la profilaxis para enfermedades oportunistas en la supervivencia de los pacientes con sida. Rev Clin Esp 2000; 200: 187-192.

54. Graham NMH, Hoover DR, Park LP, et al, for the Multicenter AIDS Cohort Study Group. Survival in HIV-infected patients who have received zidovudine: comparison of combination therapy with sequential monotherapy and continued zidovudine mootherapy. Ann Intern Med 1996; 124: 1.031-1.038.

55. Saravolatz LD, Winslow DL, Collins G, et al. Zidovudine alone or in combination with didanosine or zalcitabine in HIV-infected patients with the Acquired Immunodeficiency Syndrome or fewer than 200 CD4 cells per cubic millimeter. N Engl J Med 1996; 335: 1.099-1.106.

56. Hammer SM, Katzenstein DA, Hughes MD, et al, for the AIDS Clinical Trials Group Study 175. A trial comparing nucleoside monotherapy with combination therapy in HIV-infected adults with CD4 cell counts from 200 to 500 per cubic millimeter. N Engl J Med 1996; 335: 1.0811.090 .

57. Phillips AN, Katlama C, Barton S, et al. for the Euro-sida Study Group. Survival in 2367 zidovudine-treated patients according to use of other nucleoside analogue drugs. J Acquir Immune Defic Syndr 1998; 17: 239-244.

58. Knobel H, Serrano C, Hernández P, Pavesi M, Díez A. Aceptación, cumplimiento y tolerancia del tratamiento antirretroviral en pacientes con infección por el virus de la inmunodeficiencia humana. An Med Interna (Madrid) 1997; 14: 445-449.

59. Knobel F, Guelar A. Adherencia: cómo medirla y mejorarla. En: González-García J, Moreno S, Rubio R, editores. Infección por VIH 1999. Madrid: Ediciones Doyma; 1999: p. 221-246. 